\title{
Estudio retrospectivo sobre las diferencias entre insuficiencia cardíaca sistólica y diastólica en un registro de 1.000 pacientes del hospital Puerta de Hierro-Majadahonda-Madrid. Comparación con el Registro Nacional español de Insuficiencia Cardíaca (RICA)
}

\author{
María Luisa Martín Jiménez* y Esther Montero Hernández
}

Servicio de Medicina Interna, Hospital Puerta de Hierro Majadahonda, Madrid, España

Recibido el 18 de noviembre de 2016; aceptado el 17 de abril de 2017

Disponible en Internet el 18 de julio de 2017

\author{
PALABRAS CLAVE \\ Insuficiencia \\ cardíaca; \\ Fracción de eyección \\ del ventrículo \\ izquierdo; \\ Disfunción diastólica
}

\begin{abstract}
Resumen
Objetivo: Comparar las diferencias entre insuficiencia sistólica y diastólica en pacientes ingresados en el Hospital Puerta de Hierro Majadahonda, con diagnóstico al alta de "primer episodio de insuficiencia cardiaca". Los resultados se cotejan con el registro nacional español de insuficiencia cardiaca (RICA).

Material y método: Estudio retrospectivo que incluye a 1.000 pacientes ingresados desde octubre 2008 hasta mayo 2010. El seguimiento se realizó hasta mayo 2015.

Resultados: El 70,6\% procedían de Medicina Interna, con predominio del sexo femenino (62\%) y una mediana de 87 años de edad.

La mortalidad global fue de $69,8 \%$, mayor en pacientes con insuficiencia cardiaca-diastólica. OR 1,03 (IC 95\% 0,73-1,45).

El 59\% de los pacientes reingresaron al menos una vez; en su mayoría eran varones (61\%) e hipertensos (89,3\%).

Conclusiones: Los pacientes con insuficiencia cardiaca-diastólica ingresan preferentemente en Medicina Interna $(76,2 \%)$; la mayoría son mujeres hipertensas y de edad más avanzada que en el grupo de insuficiencia cardiaca-sistólica.

Los resultados fueron similares a los del registro RICA, salvo mayor mortalidad y reingresos, probablemente por la edad más avanzada de los pacientes y el mayor tiempo de seguimiento. (C) 2017 Sociedad Colombiana de Cardiología y Cirugía Cardiovascular. Publicado por Elsevier España, S.L.U. Este es un artículo Open Access bajo la licencia CC BY-NC-ND (http:// creativecommons.org/licenses/by-nc-nd/4.0/).
\end{abstract}

\footnotetext{
* Autor para correspondencia.

Correo electrónico: mluisamartinj@gmail.com (M.L. Martín Jiménez).
} 


\section{KEYWORDS}

Heart failure;

Left ventricular

ejection fraction;

Diastolic dysfunction
Retrospective study on the differences between systolic and diastolic heart failure in a registry of 1,000 patients from the Puerta de Hierro-Majadahonda Hospital, Madrid. Comparison with the Spanish National Heart Failure Registry

\footnotetext{
Abstract

Objective: To compare the differences between systolic and diastolic dysfunction in patients admitted to the Hospital Puerta de Hierro Majadahonda, Madrid, with a diagnosis on discharge of "first episode of heart failure". The results were compared with the Spanish National Heart Failure Registry (RICA).

Material and method: A retrospective study that included 1,000 patients admitted from October 2008 to May 2010. The follow-up was carried out up to May 2015.

Results: The majority of patients were admitted to Internal Medicine, with a majority of females $(62 \%)$, and with a median age of 87 years.

The overall mortality was $69.8 \%$, and was higher in patients with diastolic heart failure (OR: 1.03, 95\% Cl; $0.73-1.45)$.

More than half of the patients (59\%) were admitted at least once, with the majority being males $(61 \%)$ and with hypertension $(89.3 \%)$.

Conclusions: Patients with diastolic heart failure were mainly admitted to Internal Medicine (76.2\%). The majority were women with hypertension, and of a more advanced age than the systolic heart failure group.

The results were similar to the RICA, except for the higher mortality and admissions, probably due to the more advanced age of the patients, and the longer follow-up time.

(c) 2017 Sociedad Colombiana de Cardiología y Cirugía Cardiovascular. Published by Elsevier España, S.L.U. This is an open access article under the CC BY-NC-ND license (http:// creativecommons.org/licenses/by-nc-nd/4.0/).
}

\section{Introducción}

La insuficiencia cardíaca es una patología muy prevalente en España, afecta al $5 \%$ de la población ${ }^{1}$, y es cada vez más frecuente debido al envejecimiento progresivo de la misma. Constituye la primera causa de hospitalización en mayores de 65 años $^{2}$ y representa el 3\% de todos los ingresos hospitalarios. Asocia múltiples comorbilidades así como número de reingresos. En pacientes de edad avanzada, predomina la insuficiencia cardiaca con fracción de eyección del ventrículo izquierdo ( $\mathrm{FEVI}$ ) preservada; siendo más frecuente en el sexo femenino (tabla 1, fig. 1).

\section{Material y método}

Se trata de un estudio retrospectivo que incluye a 1.000 pacientes ingresados con diagnóstico al alta de "primer episodio de insuficiencia cardíaca"' en el Hospital Universitario Puerta de Hierro Majadahonda (HUPHM) desde octubre de 2008 hasta mayo de 2010. El número final de pacientes estudiados fue de 824, pues en 176 casos no se trató del primer episodio de insuficiencia cardiaca. Se hizo ecocardiograma transtorácico a 665 pacientes.

Procedían tanto de Cardiología como de Medicina Interna. El seguimiento se hizo hasta mayo de 2015, para una mediana de 21,2 meses.

Se recogen variables epidemiológicas (edad y sexo), características ecocardiográficas (FEVl; valvulopatías, hipertrofia ventricular), valor de proBNP, comorbilidades asociadas, supervivencia y tasa de reingresos. Se excluyen
Tabla 1 Comparación con el registro RICA

\begin{tabular}{lll}
\hline & HUPHM & Registro RICA \\
\hline $\begin{array}{l}\text { Número de } \\
\text { pacientes }\end{array}$ & 1.000 & 2.190 \\
$\begin{array}{l}\text { Seguimiento } \\
\quad \text { (meses) }\end{array}$ & 21,2 (mediana) & 12 \\
Edad media (años) & 87 & \\
Sexo femenino (\%) & 62 & 78,6 \\
HTA (\%) & 86,3 & 53 \\
FA (\%) & 49,9 & 85 \\
DM tipo 2 (\%) & 35,9 & 54,3 \\
Insuficiencia renal & 26,8 & 44,3 \\
$\quad$ (FG, ml/min) & & 58,2 \\
Anemia (\%) & 23,3 & 53,2 \\
EPOC (\%) & 23,3 & 27,4 \\
Reingresos (\%) & 59 & 43,2 \\
Mortalidad global & 69,8 & 26,3 \\
$\quad$ (\%) & & \\
\hline
\end{tabular}

pacientes con insuficiencia cardiaca congestiva debida a hipertensión pulmonar y diabéticos tipo 1 .

\section{Objetivo}

Comparar las diferencias en cuanto a clínica, pronóstico y comorbilidades entre ambos grupos: FEVI preservada ( $\mathrm{FEVI}>50 \%$ ) y $\mathrm{FEVI}$ disminuida (IC-s). 


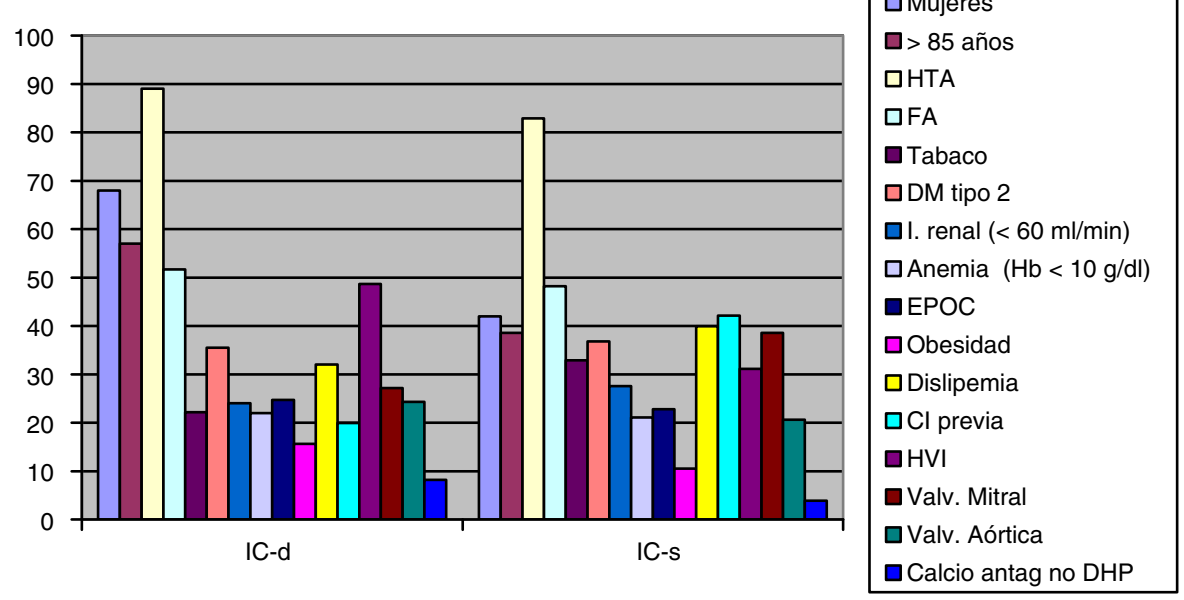

Figura 1 Principales diferencias entre insuficiencia cardiaca sistólica y diastólica.

\section{Objetivos secundarios}

Describir la mortalidad, la incidencia de reingresos y el tiempo transcurrido hasta dicho evento. El diagnóstico de insuficiencia cardíaca se realizó por la clínica, los valores de proBNP $(\mathrm{pg} / \mathrm{ml}$ ) y el ecocardiograma. Este último sólo estuvo disponible en 665 pacientes. En todos ellos fue realizado por el servicio de Cardiología del Hospital Puerta de Hierro.

Los resultados obtenidos se compararon con los del Registro nacional de insuficiencia cardíaca (RICA) del grupo de trabajo de insuficiencia cardiaca de la Sociedad española de Medicina interna (SEMI) ${ }^{3}$. Se trata de un estudio nacional de cohortes multicéntrico que incluye a 2.190 pacientes procedentes de 52 hospitales. De ellos, el $59,1 \%$ tiene insuficiencia cardiaca-diastólica, tratándose del primer episodio en 683 pacientes.

\section{Resultados}

La mayoría de los pacientes analizados procedían de Medicina interna $(70,6 \%)$. El $62 \%$ correspondió a mujeres y la mediana de edad fue de 87 años.

En cuanto a las diferencias según la FEVI, el 76,2\% de los pacientes ingresados en Medicina Interna tenían insuficiencia cardiaca-diastólica, siendo el porcentaje de esta de 23,8\% en los pacientes procedentes de Cardiología.

El valor de NTproBNP estuvo más elevado en el grupo de insuficiencia cardiaca-sistólica (mediana $6.300 \mathrm{pg} / \mathrm{ml}$ ), siendo la mediana en insuficiencia cardiaca-diastólica de $2.386 \mathrm{pg} / \mathrm{ml}(\mathrm{p}=0,087)$.

Los pacientes con insuficiencia cardiaca-diastólica ingresaron preferentemente en Medicina interna, en ellos predominó el sexo femenino, la edad más avanzada, la hipertensión arterial (HTA) con hipertrofia ventricular izquierda y el empleo de calcioantagonistas no dihidropiridínicos (DHP); todos ellos con resultados estadísticamente significativos. Es importante señalar que estos fármacos sólo se han usado en 45 pacientes del total de 1.000. También son más frecuentes en este grupo, aunque sin significación estadística, la presencia de fibrilación auricular (FA), anemia, EPOC, obesidad y valvulopatía aórtica.
En este registro llama la atención el escaso número de pacientes con obesidad (IMC $>30 \mathrm{~kg} / \mathrm{m}^{2}$ ), únicamente presente en el $12,1 \%$, ya que la prevalencia de dicha patología en la población de más de 65 años es del $35 \%^{4}$; probablemente se deba a que este dato no queda recogido en la historia clínica, pues son muchos los pacientes que no son tallados ni pesados al ingreso.

Por el contrario, el grupo de insuficiencia cardiacasistólica suele ingresar en Cardiología, siendo más frecuente el sexo masculino, el antecedente de cardiopatía isquémica en el último año, la valvulopatía mitral asociada, el tabaquismo y la dislipemia; con significación estadística. Los pacientes con insuficiencia renal y diabetes mellitus (DM) tipo 2 predominan en este grupo, aunque sin diferencias estadísticamente significativas.

La mortalidad tras una mediana de seguimiento de 21,2 meses fue del $69,8 \%$, con pérdida a lo largo del seguimiento de 16 pacientes. Es mayor en pacientes con insuficiencia cardiaca-diastólica aunque no significativa; OR 1,03 (IC 95\% 0,73-1,45).

Por el contrario, y como es de esperarse, la edad más avanzada se asocia a mayor mortalidad (OR 1,73 IC 95\% 1,402,08); también la insuficiencia renal definida como filtrado glomerular (FG) < $60 \mathrm{ml} / \mathrm{min}$ (OR 1,59 IC 95\% 1,31-1,92); la anemia con valores de hemoglobina $(\mathrm{Hb})$ inferiores a $10 \mathrm{~g} / \mathrm{dl}$ (OR 1,53 IC 95\% 1,26-1,86) y la enfermedad pulmonar obstructiva crónica (OR 1,22 IC 95\% 1,01-1,48).

El $59 \%$ de los pacientes reingresan al menos una vez, con un tiempo medio hasta el primer reingreso de 12 meses. La mayoría de los que reingresan son varones $(61 \%)$, mayores de 85 años $(53,3 \%$ ) y con HTA $(89,3 \%)$ y DM2 $(39,5 \%)$ como comorbilidades más asociadas.

\section{Discusión}

La insuficiencia cardiaca-diastólica predomina en pacientes de edad avanzada, siendo más frecuente en mujeres con HTA e hipertrofia ventricular izquierda como principales comorbilidades ${ }^{5}$. En este estudio, la coexistencia de fibrilación auricular también es más común en este grupo, aunque con resultados no significativos. Se espera que su 
prevalencia vaya en aumento debido al envejecimiento progresivo de la población, de ahí la importancia de conocer tanto su fisiopatología como las distintas opciones terapéuticas ${ }^{6,7}$. Ningún fármaco ha demostrado disminuir la morbimortalidad ${ }^{8}$, por tanto son fundamentales el control de la presión arterial y de la frecuencia cardíaca, al aumentar el tiempo de diástole y favorecer así el llenado ventricular. En este estudio llama la atención el escaso empleo de calcioantagonistas no DHP en pacientes con insuficiencia cardiaca-diastólica, únicamente en el $8,2 \%$; probablemente en relación a un mayor empleo de betabloqueadores y/o digoxina como estrategia de control de la frecuencia cardíaca.

La mortalidad de este registro es muy elevada, el 69,8\% tras siete años de seguimiento, con una supervivencia acumulada hasta mayo 2015, del 30,2\%. La edad más avanzada que en otros estudios (mediana de edad de 87 años) y el mayor tiempo de seguimiento son factores que podrían explicar este resultado. Por el contrario, a pesar de la alta tasa de mortalidad, sólo reingresan el $59 \%$ de los pacientes, mientras que el $41 \%$ restantes no lo hace, quizá porque es muy probable que pertenezcan al grupo de pacientes fallecidos, pues a lo largo del seguimiento el número de pacientes perdidos es únicamente de 16.

En cuanto a la comparación con el registro nacional de insuficiencia cardiaca de la SEMI se destaca que el registro del presente estudio incluye a 1.000 pacientes con una mediana de seguimiento de 21,2 meses, mientras que el estudio RICA incluye a más del doble de pacientes (2.190) con la mitad de tiempo de seguimiento ${ }^{3}$. La mayor prevalencia de pacientes con anemia en el registro RICA se explica porque definen dicha patología con valores de $\mathrm{Hb}$ inferiores a $12 \mathrm{~g} / \mathrm{dl}$ en mujeres y menores de $13 \mathrm{~g} / \mathrm{dl}$ en varones, mientras que en este estudio la anemia queda definida como $\mathrm{Hb}<10 \mathrm{~g} / \mathrm{dl}$.

La prevalencia de insuficiencia renal es casi el doble en el registro nacional; definida en ambos estudios como aclaramiento de creatinina inferior a $60 \mathrm{ml} / \mathrm{min}$, sin que se haya encontrado una causa que justifique esta diferencia. Por último, en el estudio del Hospital Puerta de Hierro, hay mayor tasa de reingresos (59 vs. $43,2 \%$ ) y mayor mortalidad $(69,8$ vs. $26,3 \%)$; estas diferencias pueden explicarse tanto por la edad más avanzada de los pacientes $(87$ vs. 78,6$)$ como por el mayor tiempo de seguimiento.

\section{Conclusiones}

Los pacientes con insuficiencia cardíaca con FEVI preservada ingresan principalmente en los servicios de Medicina interna, la mayoría son mujeres de edad avanzada y con más comorbilidades que los pacientes con FEVI disminuida, siendo la más prevalente la hipertensión arterial con hipertrofia ventricular izquierda asociada.

Con los resultados expuestos anteriormente se plantea la necesidad de un seguimiento multidisciplinar en los pacientes con insuficiencia cardiaca congestiva con FEVI preservada, principalmente en unidades de insuficiencia cardíaca coordinadas por especialistas en Medicina interna. De este modo, disminuirían tanto las visitas a urgencias como los ingresos por descompensación, mejorando así la calidad de vida de los pacientes.

\section{Responsabilidades éticas}

Protección de personas y animales. Los autores declaran que para esta investigación no se han realizado experimentos en seres humanos ni en animales.

Confidencialidad de los datos. Los autores declaran que han seguido los protocolos de su centro de trabajo sobre la publicación de datos de pacientes.

Derecho a la privacidad y consentimiento informado. Los autores declaran que en este artículo no aparecen datos de pacientes.

\section{Conflicto de intereses}

Los autores declaran no tener ningún conflicto de intereses.

\section{Agradecimientos}

A Elena García Guijarro, Juan Francisco Montoro Lara, Marta Vivas Sanz, Carmen Velasco Tejado, Manuel Fernández Tomás, Camino Gómez Peñalba, Agustín Albarracín Serra, Isabel Millán Santos, Luis Antonio Alonso-Pulpó y Valentín Cuervas-Mons Martínez.

\section{Bibliografía}

1. Sayago-Silva I, García F, Segovia-Cubero J. Epidemiología de la insuficiencia cardiaca en España en los últimos 20 años. Rev Esp Cardiol. 2013;66:649-56.

2. Conde-Martel A, Arkuch ME, Formiga F, Manzano-Espinosa L, Aramburu, Bodas O, González-Franco A, et al. Diferencias en función del sexo en el perfil.clínico y pronóstico de pacientes con insuficiencia cardíaca. Resultados del.Registro RICA. Rev Clin Esp. 2015. Disponible en: http://dx.doi.org/10.1016/j.rce.2015.02.010.

3. Franco J, Formiga F, Chivite D, Manzano L, Carrera M, ArévaloLorido JC. New onset heart failure - clinical characteristics and short-term mortality. A RICA (Spanish registry of acute heart failure) study. Eur J Int Med. 2015;26:357-62.

4. Aranceta-Bartrina J, Serra-Majem L, Foz-Sala M, Moreno-Esteban B. y grupo.colaborativo SEEDO. Prevalencia de obesidad en España. Med Clin (Barc). 2005;125:460-6.

5. Borlaug BA. Defining HFpEF: where do we draw the line? Eur Heart.J. doi:10.1093/eurheartj/ehv561.

6. 2016 ESC Guidelines for the diagnosis and treatment of acute and chronic heart failure. Eur Heart J. doi:10.1093/eurheartj/ehw128.

7. Redfield MM. Heart failure with preserved ejection fraction. N Engl J Med. 2016;375:19.

8. Vaduganathan M, Michel A, Hall K, Mulligan C, Nodari S, Shan $S$, et al. Spectrum of epidemiological and clinical findings in patients with heart failure.preserved ejection fraction stratified by study design: a systematic review. Eur J Heart Fail. 2015, http://dx.doi.org/10.1002/ejhf.442. 